\title{
A Heuristic Sequencing Method for Time Optimal Tracking of Open and Closed Paths
}

\author{
Christian Zauner ${ }^{1}$, Hubert Gattringer ${ }^{1}$, Andreas Müller ${ }^{1}$, Matthias Jörgl ${ }^{2}$ \\ ${ }^{1}$ Institute of Robotics \\ Johannes Kepler University Linz \\ Altenbergerstraße 69, 4040 Linz, Austria \\ [christian.zauner, hubert.gattringer, a.mueller]@jku.at \\ 2 Trotec Laser GmbH \\ Freilingerstraße 99, 4614 Marchtrenk, Austria \\ matthias.joergl@troteclaser.com
}

\begin{abstract}
Tracking sequences of predefined open and closed paths is of crucial interest for applications like laser cutting and similar production processes. These distinct paths are connected by non-productive, four times continuously differentiable trajectories, which also account for the overall process time. Heuristic methods are applied in order to find a proper sequencing of the open and closed path and thereby minimize the overall process time subject to constraints given by the system limits. To this end the exact traversing times of the non-productive linking trajectories are computed, which also have to be time optimal subject to the system limits. Finally two heuristic algorithms are presented and compared with respect to solution quality and calculation time using randomly generated problems.
\end{abstract}

Keywords: Path Planning, Heuristic Scheduling, Traveling Salesman Problem, Laser Cutting Machine.

\section{INTRODUCTION}

For laser cutting applications a cutting job consists of predefined open and closed paths. Since theses paths are often not connected the question arises, how these paths should be sorted in order to minimize overall process time. Numerous contributions, concerning this topic, can be found in the literature, as indicated by the review paper [1], where 72 contributions are collected and compared with respect to the used methods and algorithms.

Although a more general approach would be conceivable, this work focuses on paths on the 2D plane and gantry like robotic systems as shown in figure 1 . These systems are subject to restrictions like maximum velocity, acceleration and jerk for each axis respectively. Furthermore there are process specific constraints. In case of the laser cutting process the maximum velocity tangentially to the path depends on the material which has to be cut and in order to ensure a clean cut the velocity at the beginning and at the end of each path has to be zero. Subject to these constraints a time optimal path tracking solution for each path can be found and the optimal partial solutions can be connected by time optimal trajectories along straight lines. With an increasing number of paths to track the impact of the non-productive traversing time introduced by these links on the overall process time is getting dominant if the sequencing is not handled properly. To this end two heuristic approaches are provided, tested and compared with respect to an integer linear programming algorithm. The optimal traversing time of the non-productive linking trajectories depends non-linearly either on the end points as well as the system limits. In contrast to e.g. [2], where a piecewise linear function is introduce approximating this non-linear behaviour, in this work analytic, non-linear expressions are derived and used for the optimal traversing time. 


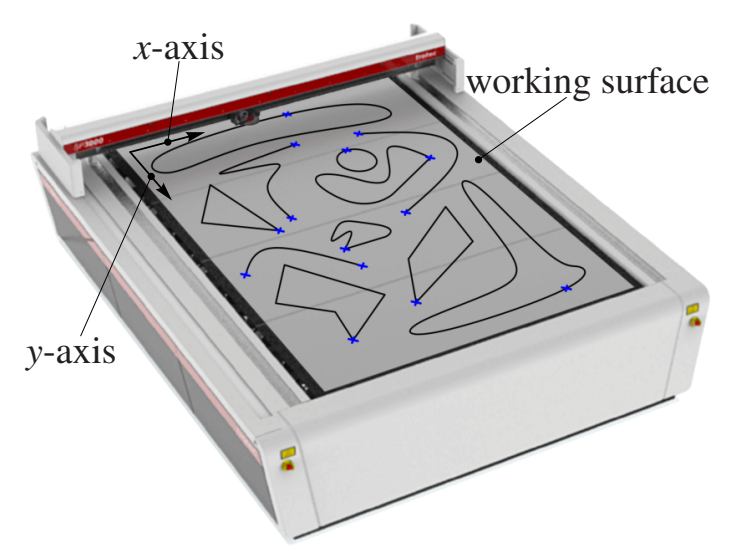

Figure 1. Gantry laser cutting machine

\section{PROBLEM DEFINITION AND COMBINATORIAL BACKGROUND}

Before setting up the problem definition some graph theoretical terminology should be introduced, which is used through out the following sections. An undirected graph is defined by a set of nodes $\mathfrak{N}=\left\{n_{i} \mid 0 \leq i<N\right\}$, where $N$ denotes the number of nodes, and a set of edges $\mathfrak{E} \subseteq\left\{e_{i, j} \mid 0 \leq\right.$ $i<N, i<j<N\}$ connecting some or all of these nodes, where edge $e_{i, j}$ is equivalent to $e_{j, i}$. An undirected graph can be

- weighted, if each edge $e_{i, j}$ is associated with a weight $w_{i, j}$.

- simple, if each edge $e_{i, j}$ is unique in $\mathfrak{E}$.

- an undirected multigraph, if edges are allowed to be not unique in $\mathfrak{E}$.

- connected, if each pair of nodes can be connected by a sequence of edges in $\mathfrak{E}$

- complete, if each pair of nodes is connected by exactly one edge.

A cycle in the graph is a sequence of edges, which starts and ends at the same node. Special cycles are Eulerian cycles, which contain every edge of a graph once, and Hamilton cycles, which start and end at the same node and traverse every other node of the graph exactly once. A Hamilton cycle is also called a tour of the graph.

A spanning tree of a connected graph is a subset of edges, which does not contain cycles and contains all nodes. Consequently the minimum spanning tree of a connected, weighted graph is the spanning tree with the minimum total edge weight.

Finding the tour with the minimum total edge weight in a complete, undirected, weighted graph is equivalent to solving the corresponding symmetric traveling salesman problem. This very well know NP-hard combinatorial problem is assumed to be not solvable in polynomial time. The number of all tours in such a graph can be stated by $N_{T}=\frac{(N-1) !}{2}$, which grows extremely fast by an increasing number of nodes. Therefore a brute force approach can only be suitable for a very low number of nodes. Although there exist many exact algorithms to solve a traveling salesman problem, like branch-and-cut or branch-and-bound, an exact solution is getting more and more impractical with an increasing number of nodes. Actually for most applications a good approximation of the optimal solution would suffice. This can be achieved efficiently by heuristic algorithms.

A cutting job, which has to be processed by the laser cutting machine, consists of several cutting paths, denoted $c_{i}$, with $1 \leq i \leq N_{c}$. Each of these paths $c_{i}$ is defined by two points in the 2D plane, a start point $\mathbf{r}_{s, i}$ and an end point $\mathbf{r}_{e, i}$. These two points are connected by a path, for which it is assumed, that a time optimal trajectory, with respect to the machine and process constraints, 
is known. Additionally a job should start and end at a defined idle position given by $\mathbf{r}_{0}$. Since the trajectories for each cutting path are already assumed to be optimal, the overall processing time can be reduced by finding a processing sequence of these paths, which minimizes the total non-productive traversing time between the consecutive paths. To this end a complete, undirected, weighted graph of the start and end points of the cutting paths is constructed with edge weights according the traversing time between these points. The optimal solution of the problem corresponds then to the solution of the symmetric traveling salesman problem given by this graph.

The cutting paths $c_{i}$ can be closed, i.e. $\mathbf{r}_{s, i}=\mathbf{r}_{e, i}$, or open, i.e. $\mathbf{r}_{s, i} \neq \mathbf{r}_{e, i}$. Without loss of generality it is assumed, that the first $N_{c c}$ paths are closed and the remaining $N_{c o}$ paths are open, which means that the conditions

$$
\begin{array}{ll}
\mathbf{r}_{s, i}=\mathbf{r}_{e, i} & \text { for } \quad 1 \leq i \leq N_{c c} \\
\mathbf{r}_{s, i} \neq \mathbf{r}_{e, i} & \text { for } \quad N_{c c}<i \leq N_{c}=N_{c c}+N_{c o}
\end{array}
$$

are fulfilled. This can be always obtained by reordering indices. Since the start and end point coincide, closed paths can be represented by a single node in the graph. Based on this, the $N=$ $N_{c c}+2 N_{c o}+1$ nodes of the graph can be associated with the start (and end) points of the cutting paths as well as the idle position according to the mapping

$$
\mathbf{r}_{n}\left(n_{i}\right)=\left\{\begin{array}{lll}
\mathbf{r}_{0} & & \text { for } i=0 \\
\mathbf{r}_{s, i} & \text { for } 1 \leq i \leq N_{c c} \\
\mathbf{r}_{s, j} \text { with } j=N_{c c}+\left(i-N_{c c}+1\right) / 2 & \text { for } \quad N_{c c}<i<N \wedge\left(i-N_{c c}\right) \text { is odd } \\
\mathbf{r}_{e, j} & \text { with } \quad j=N_{c c}+\left(i-N_{c c}\right) / 2 & \text { for } \quad N_{c c}<i<N \wedge\left(i-N_{c c}\right) \text { is even }
\end{array} .\right.
$$

With the definition of the edge weights, provided in the following section, the graph is fully defined and the traveling salesman problem can be solved. But if open cutting paths are present, i.e. $N_{c o}>0$, each algorithm used has to enforce, that these paths are actually traversed in the solution.

\section{OBJECTIVE FUNCTION}

Regardless of whether the problem is solved by an exact algorithm or by a heuristic, the objective is to find the tour with the minimum total edge weight. In order to compute the edge weights, a special metric is introduced. Since the overall goal is to achieve a time optimal solution all the considered links connecting two points on the 2D plane have to be time optimal on their own. Therefore the distance between two arbitrary points can be expressed by the minimum time needed to traverse a straight line between these two points satisfying the constraints of the robotic system. The resulting distance measure satisfies the triangle inequality as well as the remaining requirements of a metric.

The transition from one point in the 2D plane $\mathbf{r}_{n}\left(n_{i}\right)=\mathbf{r}_{i}=\left[\begin{array}{ll}x_{i} & y_{i}\end{array}\right]^{\top}$ to another point $\mathbf{r}_{n}\left(n_{j}\right)=$ $\mathbf{r}_{j}=\left[\begin{array}{ll}x_{j} & y_{j}\end{array}\right]^{\top}$, corresponding to the nodes $n_{i}$ and $n_{j}$ respectively, is performed according to a time optimal $\sin ^{2}$-jerk trajectory. This trajectory has the property, that it is continuously differentiable until the fourth derivative, which is beneficial, since the excitation of vibrations is reduced. Another advantage of this trajectory is, that the minimum traversing time between two points can be calculated analytically with respect to maximum velocity, acceleration and jerk. These maximum values can be defined for the each axis individually by $v_{\max , k}, a_{\max , k}$ and $j_{\max , k}$ with $k \in\{x, y\}$. Additionally the maximum tangential velocity, acceleration and jerk can be constrained by $v_{\max , t}$, $a_{\text {max }, t}$ and $j_{\text {max }, t}$ respectively. In order to calculate the minimum traversing time the distances $\Delta x_{i, j}=x_{j}-x_{i}$ and $\Delta y_{i, j}=y_{j}-y_{i}$ along each axis are needed, which can be used to calculate the Euclidean distance

$$
\Delta d_{i, j}=\sqrt{\Delta x_{i, j}^{2}+\Delta y_{i, j}^{2}}
$$


between the two points. With these distance measures the actual maximum values for a straight path between the points $\mathbf{r}_{i}$ and $\mathbf{r}_{j}$ can be stated by

$$
\begin{aligned}
& v_{\max , i, j}=\min \left(v_{\max , x} \frac{\Delta d_{i, j}}{\left|\Delta x_{i, j}\right|}, v_{\max , y} \frac{\Delta d_{i, j}}{\left|\Delta y_{i, j}\right|}, v_{\max , t}\right), \\
& a_{\text {max }, i, j}=\min \left(a_{\max , x} \frac{\Delta d_{i, j}}{\left|\Delta x_{i, j}\right|}, a_{\max , y} \frac{\Delta d_{i, j}}{\left|\Delta y_{i, j}\right|}, a_{\max , t}\right), \\
& j_{\text {max }, i, j}=\min \left(j_{\max , x} \frac{\Delta d_{i, j}}{\left|\Delta x_{i, j}\right|}, j_{\max , y} \frac{\Delta d_{i, j}}{\left|\Delta y_{i, j}\right|}, j_{\max , t}\right) .
\end{aligned}
$$

The trajectory over time $t$ can then be defined by

$$
\mathbf{r}_{i, j}(t)=\mathbf{r}_{i}+\frac{\mathbf{r}_{j}-\mathbf{r}_{i}}{\Delta d_{i, j}} \xi\left(t, \Delta d_{i, j}, v_{\max , i, j}, a_{\max , i, j}, j_{\max , i, j}\right)
$$

with the function $\xi\left(t, \Delta d, v_{\max }, a_{\max }, j_{\max }\right)$ satisfying the properties

$$
\begin{aligned}
& \xi\left(0, \Delta d, v_{\max }, a_{\max }, j_{\max }\right)=0, \\
& \xi\left(t_{E}, \Delta d, v_{\max }, a_{\max }, j_{\max }\right)=\Delta d, \\
& 0 \leq \dot{\xi}\left(t, \Delta d, v_{\max }, a_{\max }, j_{\max }\right) \leq v_{\max },
\end{aligned}
$$

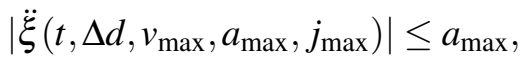

$$
\begin{aligned}
& \left|\dddot{\xi}\left(t, \Delta d, v_{\max }, a_{\max }, j_{\max }\right)\right| \leq j_{\max } .
\end{aligned}
$$

By partitioning the traversing time $t_{E}$ in 7 phases by interleaving 4 jerk phases of length $t_{j}$ with 3 jerk free phases of lengths $t_{a}, t_{v}$ and $t_{a}$ corresponding to phases of constant acceleration respectively velocity, $\xi(t)$ and its derivatives (omitting the last arguments for brevity) can be stated by

$$
\begin{aligned}
& \dddot{\xi}(t)= \begin{cases}0 & t<0 \\
j_{\max } \sin \left(\frac{\pi t}{t_{j}}\right)^{2} & 0 \leq t<t_{j} \\
0 & t_{j} \leq t<t_{j}+t_{a} \\
-j_{\max } \sin \left(\frac{\pi\left(t-t_{j}-t_{a}\right)}{t_{j}}\right)^{2} & \begin{array}{l}
t_{j}+t_{a} \leq t<2 t_{j}+t_{a} \\
0
\end{array} \\
\dddot{2} t_{j}+t_{a} \leq t \leq \frac{t_{E}}{2} \\
\frac{t_{E}}{2}<t\end{cases} \\
& \ddot{\xi}\left(t_{E}-t\right) \\
& \dot{\xi}(t)=\int_{0}^{t} \dddot{\xi}(\tau) \mathrm{d} \tau, \quad \int_{0}^{t} \ddot{\xi}(\tau) \mathrm{d} \tau, \quad \xi(t)=\int_{0}^{t} \dot{\xi}(\tau) \mathrm{d} \tau
\end{aligned}
$$

with $t_{E}=4 t_{j}+2 t_{a}+t_{v}, t_{j}=0$ if $\Delta d=0, t_{j}>0$ if $\Delta d>0, t_{a} \geq 0$ and $t_{v} \geq 0$. The maximum values of $\ddot{\xi}(t)$ and $\dot{\xi}(t)$ as well as the final value $\xi\left(t_{E}\right)$ can be expressed in terms of

$$
\begin{aligned}
\max _{t}(|\ddot{\xi}(t)|) & =\ddot{\xi}\left(t_{j}\right)=\frac{j_{\max } t_{j}}{2}=\hat{a} \leq a_{\max }, \\
\max _{t}(|\dot{\xi}(t)|) & =\dot{\xi}\left(2 t_{j}+t_{a}\right)=\hat{a} t_{a}+\frac{2 \hat{a}^{2}}{j_{\max }}=\hat{v} \leq v_{\max }, \\
\xi\left(t_{E}\right) & =\hat{a} t_{a}{ }^{2}+\frac{6 \hat{a}^{2} t_{a}}{j_{\max }}+\hat{v} t_{v}+\frac{8 \hat{a}^{3}}{j_{\max }{ }^{2}}=\Delta d .
\end{aligned}
$$

In order to get a time optimal trajectory, four cases have to be distinguished, which have the following solutions: 
- $t_{a}=0 \wedge \hat{a} \leq a_{\max } \wedge t_{v}=0 \wedge \hat{v} \leq v_{\max }$

$$
\begin{gathered}
\hat{a}=\frac{1}{2} \sqrt[3]{j_{\max }^{2} \Delta d} \quad \hat{v}=\frac{1}{2} \sqrt[3]{j_{\max } \Delta d^{2}} \quad t_{j}=\sqrt[3]{\frac{\Delta d}{j_{\max }}} \\
\Delta d \leq \sqrt{\frac{8 v_{\max }^{3}}{j_{\max }}} \wedge v_{\max } \leq \frac{2 a_{\max }^{2}}{j_{\max }} \vee \Delta d \leq \frac{8 a_{\max }^{3}}{j_{\max }^{2}} \wedge v_{\max }>\frac{2 a_{\max }^{2}}{j_{\max }}
\end{gathered}
$$

- $t_{a}=0 \wedge \hat{a} \leq a_{\max } \wedge t_{v}>0 \wedge \hat{v}=v_{\max }$

$$
\begin{gathered}
\hat{a}=\sqrt{\frac{j_{\max } v_{\max }}{2}} \quad t_{v}=\frac{\Delta d}{v_{\max }}-\sqrt{\frac{8 v_{\max }}{j_{\max }}} \quad t_{j}=\sqrt{\frac{2 v_{\max }}{j_{\max }}} \\
\Delta d>\sqrt{\frac{8 v_{\max }^{3}}{j_{\max }}} \wedge v_{\max } \leq \frac{2 a_{\max }^{2}}{j_{\max }}
\end{gathered}
$$

- $t_{a}>0 \wedge \hat{a}=a_{\max } \wedge t_{v}=0 \wedge \hat{v} \leq v_{\max }$

$$
\begin{gathered}
\hat{v}=\sqrt{\frac{a_{\max }^{4}}{j_{\max }^{2}}+a_{\max } \Delta d}-\frac{a_{\max }^{2}}{j_{\max }} \quad t_{a}=\sqrt{\frac{a_{\max }^{2}}{j_{\max }^{2}}+\frac{\Delta d}{a_{\max }}}-\frac{3 a_{\max }}{j_{\max }} \quad t_{j}=\frac{2 a_{\max }}{j_{\max }} \\
\frac{8 a_{\max }^{3}}{j_{\max }^{2}}<\Delta d \leq \frac{2 v_{\max } a_{\max }}{j_{\max }}+\frac{v_{\max }^{2}}{a_{\max }} \Longrightarrow v_{\max }>\frac{2 a_{\max }{ }^{2}}{j_{\max }}
\end{gathered}
$$

- $t_{a}>0 \wedge \hat{a}=a_{\max } \wedge t_{v}>0 \wedge \hat{v}=v_{\max }$

$$
\begin{gathered}
t_{a}=\frac{v_{\max }}{a_{\max }}-\frac{2 a_{\max }}{j_{\max }} \quad t_{v}=\frac{\Delta d}{v_{\max }}-\frac{v_{\max }}{a_{\max }}-\frac{2 a_{\max }}{j_{\max }} \quad t_{j}=\frac{2 a_{\max }}{j_{\max }} \\
\Delta d>\frac{2 v_{\max } a_{\max }}{j_{\max }}+\frac{v_{\max }^{2}}{a_{\max }} \wedge v_{\max }>\frac{2 a_{\max }^{2}}{j_{\max }}
\end{gathered}
$$

The terminal time of $\xi\left(t, \Delta d, v_{\max }, a_{\max }, j_{\max }\right)$ can then be stated by

$$
\begin{aligned}
& t_{E}\left(\Delta d, v_{\max }, a_{\max }, j_{\max }\right)= \\
& \left\{\begin{array}{ll}
4 \sqrt[3]{\frac{\Delta d}{j_{\max }}} & \Delta d \leq \sqrt{\frac{8 v_{\max }{ }^{3}}{j_{\max }}} \wedge \Delta d \leq \frac{8 a_{\max }{ }^{3}}{j_{\max }^{2}} \\
\sqrt{\frac{8 v_{\max }}{j_{\max }}}+\frac{\Delta d}{v_{\max }} & \Delta d>\sqrt{\frac{8 v_{\max }{ }^{3}}{j_{\max }}} \wedge v_{\max } \leq \frac{2 a_{\max }^{2}}{j_{\max }} \\
2\left(\frac{a_{\max }}{j_{\max }}+\sqrt{\frac{a_{\max }{ }^{2}}{j_{\max }^{2}}+\frac{\Delta d}{a_{\max }}}\right) & \frac{8 a_{\max }{ }^{2}}{j_{\max }{ }^{2}}<\Delta d \leq \frac{2 v_{\max } a_{\max }}{j_{\max }}+\frac{v_{\max }{ }^{2}}{a_{\max }} \\
\frac{2 a_{\max }}{j_{\max }}+\frac{v_{\max }}{a_{\max }}+\frac{\Delta d}{v_{\max }} & \Delta d>\frac{2 v_{\max } a_{\max }}{j_{\max }}+\frac{v_{\max }}{a_{\max }} \wedge v_{\max }>\frac{2 a_{\max }{ }^{2}}{j_{\max }}
\end{array} .\right.
\end{aligned}
$$

Finally the minimum traversing time between the points $\mathbf{r}_{i}$ and $\mathbf{r}_{j}$, which defines the edge weight $w_{i, j}$ between the nodes $n_{i}$ and $n_{j}$, is

$$
w_{i, j}=t_{E}\left(\Delta d_{i, j}, v_{\max , i, j}, a_{\max , i, j}, j_{\max , i, j}\right) .
$$

\section{CHRISTOFIDES ALGORITHM}

The Christofides algorithm [3] is a construction heuristic based on the minimum spanning tree of the complete, undirected, weighted graph. The main steps of the algorithm are as follows:

- Build the minimum spanning tree 
- Select all nodes of the minimum spanning tree with an odd degree

- Find a minimum-weight perfect matching of the complete subgraph of these nodes

- Combine the edges from the perfect matching with the minimum spanning tree

- Form an Eulerian cycle on the result

- Convert the Eulerian cycle to a Hamiltonian circle (tour) by skipping all repeating nodes

The total sum of the edge weights (tour length) of the so gained tour over all nodes of the graph is guaranteed to be within 1 and 1.5 times the length of the optimal tour, if the edge weights satisfy the triangle inequality.

As shown in [4] this algorithm can also be used to obtain a near optimal solution for problems with $N_{c o}>0$, i.e. open paths are present. In order to guarantee that an open path $c_{j}$ with $j>N_{c c}$ with the corresponding nodes $n_{i}$ and $n_{i+1}$, according to start and end point thereof, is traversed, the weight $w_{i, i+1}$ has to be modified according to

$$
w_{i, i+1}=\min \left(\min \left\{w_{i, k} \mid k>i+1\right\}, \min \left\{w_{k, i+1} \mid k<i\right\}\right) .
$$

This ensures, that the edge corresponding to an open cutting path is selected, when building the minimum spanning tree. Additionally it has to be ensured, that these edges are not skipped when converting the Eulerian cycle to the Hamilton cycle. As stated in [4] this procedure leads to the downside that the triangle inequality is no longer satisfied and the major benefit, which is the upper bound of 1.5 times of the optimal tour length, does no longer apply.

The algorithm used for solving the examples is implemented in Python based on the packages numpy and networkx. In order to speed up the calculation time of the minimum-weight perfect matching a heuristic approach is chosen for problems with $N \geq 1000$. Therefore the minimumweight perfect matching is not performed on the complete subgraph of the odd degree nodes, but on a minimum-weight sparse subgraph thereof, with a minimum degree of the occurring nodes of five.

\section{LIN-KERNIGHAN-HELSGAUN (LKH) ALGORITHM FOR PROBLEMS WITH OPEN AND CLOSED PATH}

In contrast to the Christofides algorithm, which terminates once a feasible solution is found, the Lin-Kernighan-Helsgaun algorithm [5], which is based on the algorithm of Lin-Kernighan [6], is an iteratively improving heuristic. After a preprocessing phase, essentially based on an extended minimum spanning tree, a suboptimal initial solution is generated with any suitable and fast construction heuristic. This initial solution is then improved by so called $k$-opt exchanges, which means that in every iteration step, $k$ edges of the current tour are replaced by $k$ other edges, in order to improve the tour. Special heuristic rules are applied to decide which edges should be removed and which edges should be used instead. This drastically reduces the according search spaces and consequently also the calculation time. To decrease the calculation time even further the $k$-opt exchanges are constructed sequentially from $k=2$ to $k=5$. Once an improvement is found the exchange is applied immediately and the algorithm proceeds with the next iteration step. The algorithm terminates when no further improvement of the tour length with respect to $k \leq 5$ and the applied heuristic rules can be found.

This algorithm can be applied straightforwardly to a problem with solely closed paths, i.e. problems with $N_{c c}>0$ and $N_{c o}=0$. In order to use this algorithm with open and closed paths, i.e. problems with $N_{c c} \geq 0$ and $N_{c o}>0$, some modifications in the problem setup and the algorithm are necessary.

In the preprocessing phase of the algorithm constructive sets are associated to each node, which are used to decide which edge should be added in the iteration phase. With open cutting paths 
present, it has to be ensured, that the according edges are part of these sets. Additionally when constructing the initial tour, it has to be ensured, that these edges are part of the initial tour. In the iterative improving phase two modifications of the algorithm are applied, in order to ensure the validity of the solution tour and to reduce the calculation time. Firstly edges according to the open cutting paths are never removed from the current solution. Secondly each time an edge $e_{i, j}$ is considered to be added to the current solution, it is determined whether the start or end node $n_{i}$ or $n_{j}$ of this edge is either start or end node of an open cutting path. If so, it is checked whether flipping the sequential order of the two end points of the affected open path may further improve the current solution. Figure 2 shows the edges (dashed lines), which are taken into account, when any edge connecting the two open paths in the figure is considered to be added to the current solution, and the edges (fully drawn lines), which are actually chosen. The algorithm used for solving the examples is implemented in $\mathrm{C}++17$ based on the standard library.

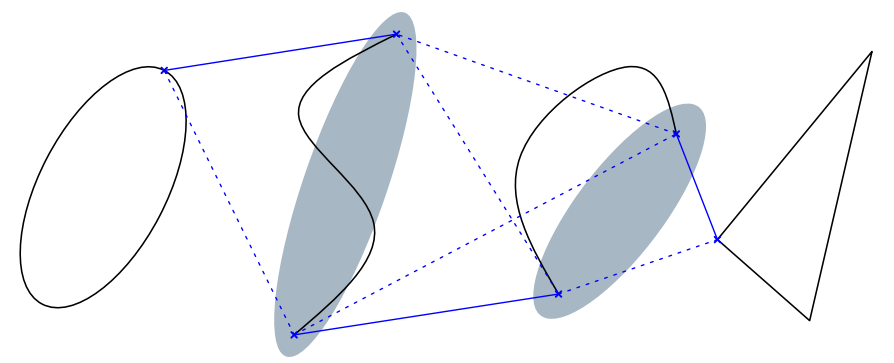

Figure 2. Selection of actually added edges in case of open paths

\section{INTEGER LINEAR PROGRAMMING}

In order to solve the traveling salesman problem by integer linear programming each edge $e_{i, j}$ is associated with a boolean variable $\hat{e}_{i, j} \in\{0,1\}$, which indicates whether the respective edge is part of the solution tour or not. According to the work of [7] the optimization problem can be stated by

$$
\begin{array}{rlr}
\min _{\hat{e}_{i, j}} & \sum_{i=0}^{N-1} \sum_{j=i+1}^{N-1} \hat{e}_{i, j} w_{i, j} & \\
\text { s.t. } & \sum_{j=i+1}^{N-1} \hat{e}_{i, j}+\sum_{j=i+1}^{N-1} \hat{e}_{j, i}=2 & 0 \leq i<N \\
& \hat{e}_{i, i+1}=1 & i \in\left\{N_{c c}+1, N_{c c}+3, \ldots, N-2\right\}
\end{array}
$$

By the first constraint it is enforced, that each node is part of the solution and has a degree of two, which means it has one incoming and one outgoing edge. The second constraint (32) ensures, that the edges associated with the open cutting paths are part of the solution edges. Solving this problem does not necessarily result in a valid tour, since also a number of cycles containing all nodes fulfil the constraints. Therefore additional constraints, which eliminate cycles have to be added. The number of this constraints grows exponentially with an increasing number of nodes. Therefore the optimization problem is solved iteratively and in every iteration constraints

$$
\sum_{\left.(i, j) \in \mathfrak{C}_{k}\right)} \hat{e}_{i, j} \leq\left|\mathfrak{C}_{k}\right|-1
$$

for each occurring cycle $\mathfrak{C}_{k}=\left\{\left(i_{1}, j_{1}\right),\left(i_{2}, j_{2}\right), \ldots,\left(i_{N_{k}}, j_{N_{k}}\right)\right\}$ are added with $N_{k}=\left|\mathfrak{C}_{k}\right|$ according to the set of $N_{k}$ edges $\left\{e_{i_{1}, j_{1}}, e_{i_{2}, j_{2}}, \ldots, e_{i_{N_{k}}, j_{N_{k}}}\right\}$ forming the cycle. The algorithm used for solving the examples is implemented in Matlab based on intlinprog $(\ldots)$.

\section{EXAMPLES}

The examples shown in this section are created randomly based on the Voronoi cells of randomly distributed points in the $2 \mathrm{D}$ plane. Each problem consist of $N_{c c}$ closed and $N_{c o}$ open cutting 
paths. The constraining maximum values use for calculating the edge weights are stated in table 1 . Table 2 compares the total edge weight of the solution tours, excluded the weights of the edges according to the open cutting paths, acquired by the different algorithms. Since all the provided algorithms are implemented in different frameworks, comparing the actual calculation time is not really meaningful. Nonetheless the approximate calculation times are stated in table 3 for reference purpose. For the problem sizes of $N>101$ the integer linear programming approach did not terminate in a reasonable time span, which is noted by a - in the tables. Graphical representations are provided for the problems of sizes $N \leq 101$, as can be seen in the figures 3 to 10 . The figures for the problems with a higher number of nodes are omitted, since the graphical representation is getting less and less expressive.

As can be seen in table 3 the Christofides heuristic and LKH heuristic perform equally well for small problem sizes, regarding solution quality as well as calculation time. But with an increasing number of nodes the LKH outperforms the Christofides heuristic in both criteria, especially regarding the calculation time. Additionally it has to be noted, that for the Christofides heuristic and problems with $N \geq 1000$, a heuristic approach has to be used for the minimum-weight perfect matching, in order to get a result in reasonable time. Remarkable is the fact, that for all the examples, for which an optimal solution has been found by the integer linear programming algorithm, the LKH heuristic leads to the exact same solution.

Table 1. Examples: Used maximum values according to the system limits

\begin{tabular}{|r||r|r|r|}
\hline & \multicolumn{1}{|c|}{$v_{\max }$} & \multicolumn{1}{c|}{$a_{\max }$} & $j_{\max }$ \\
\cline { 2 - 4 } & $\mathrm{ms}^{-1}$ & $\mathrm{~m} \mathrm{~s}^{-2}$ & $\mathrm{~m} \mathrm{~s}^{-3}$ \\
\hline \hline$x$-axis & 1 & 5 & 50 \\
\hline$y$-axis & 1 & 5 & 50 \\
\hline tangentially & 1.2 & $\infty$ & $\infty$ \\
\hline
\end{tabular}

Table 2. Examples: Total edge weight of solution tour for different algorithms

\begin{tabular}{|r|r|r||r|r|r|r|}
\hline \multicolumn{3}{|c||}{ Problem sizes } & \multicolumn{3}{c|}{ Total edge weight of solution tour in s } \\
\hline$N$ & $N_{c c}$ & $N_{c o}$ & Unsorted & LKH & Christofides & ILP \\
\hline \hline 31 & 25 & 5 & 62.470 & 23.931 & 24.282 & 23.931 \\
\hline 101 & 70 & 30 & 163.539 & 58.215 & 60.881 & 58.215 \\
\hline 301 & 250 & 50 & 506.788 & 145.305 & 151.549 & - \\
\hline 1001 & 700 & 300 & 1675.920 & 378.299 & 399.266 & - \\
\hline 3001 & 2500 & 500 & 4975.530 & 976.974 & 1020.000 & - \\
\hline
\end{tabular}

Table 3. Examples: Approximate calculation time for different algorithms

\begin{tabular}{|r|r|r||r|r|r|r|}
\hline \multicolumn{3}{|c||}{ Problem sizes } & \multicolumn{5}{c|}{ Approximate calculation time in s } \\
\hline$N$ & $N_{c c}$ & $N_{c o}$ & Edge Weights & LKH & Christofides & ILP \\
\hline \hline 31 & 25 & 5 & $3.5 \times 10^{-2}$ & $3.8 \times 10^{-1}$ & $4.1 \times 10^{-1}$ & 2.9 \\
\hline 101 & 70 & 30 & $4.3 \times 10^{-2}$ & 1.9 & 2.3 & 11.2 \\
\hline 301 & 250 & 50 & $1.0 \times 10^{-1}$ & 3.6 & 27.6 & - \\
\hline 1001 & 700 & 300 & $4.8 \times 10^{-1}$ & 11.7 & 28.5 & - \\
\hline 3001 & 2500 & 500 & 1.0 & 19.9 & 213 & - \\
\hline
\end{tabular}




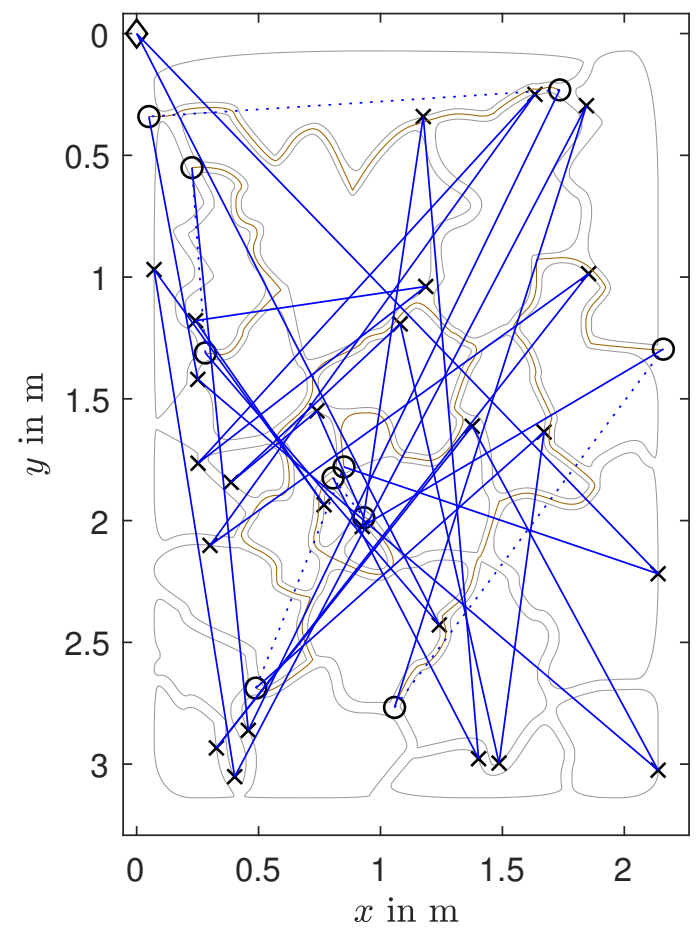

$\times$ Closed O Open - Unsorted

Figure 3. Example $N=31$ : Random Tour

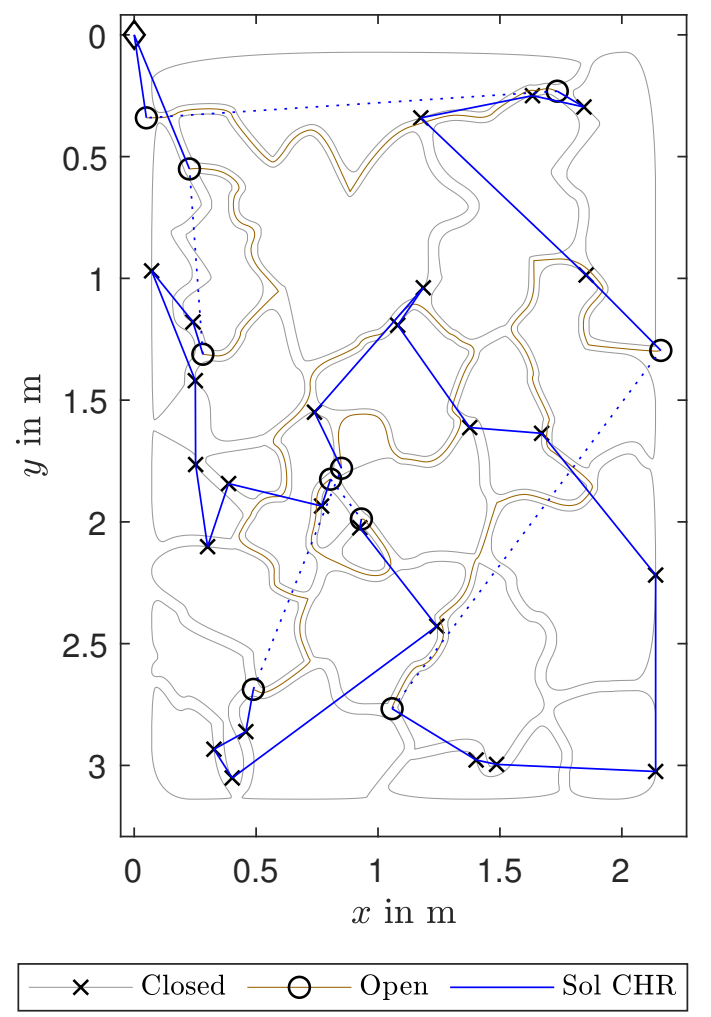

Figure 5. Example $N=31$ : Solution Christofides

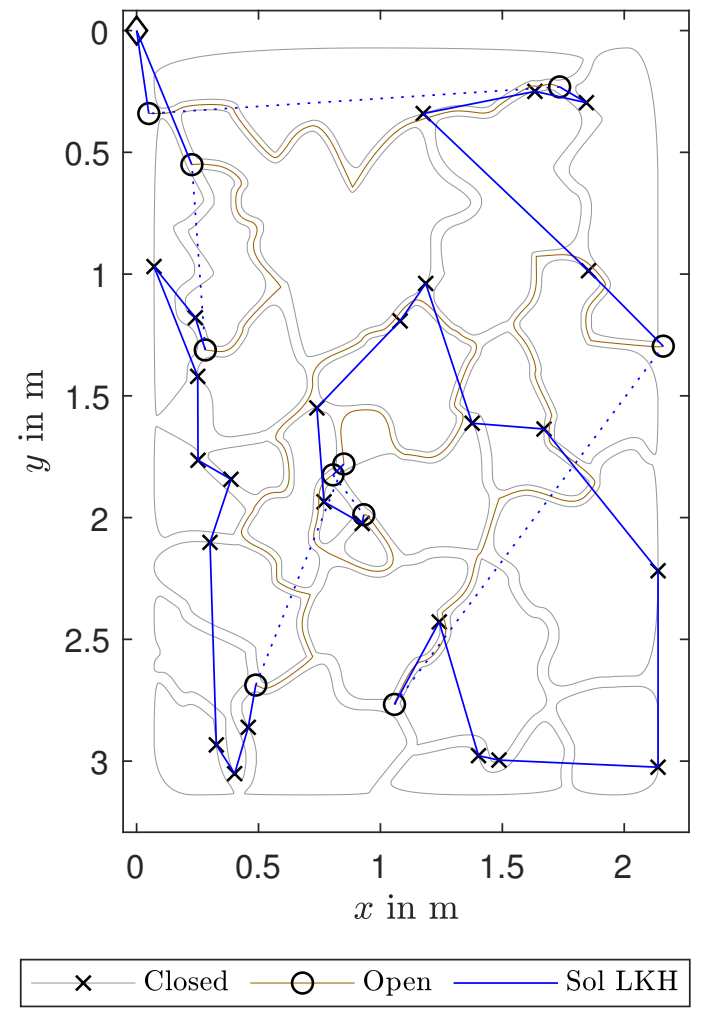

Figure 4. Example $N=31$ : Solution LKH

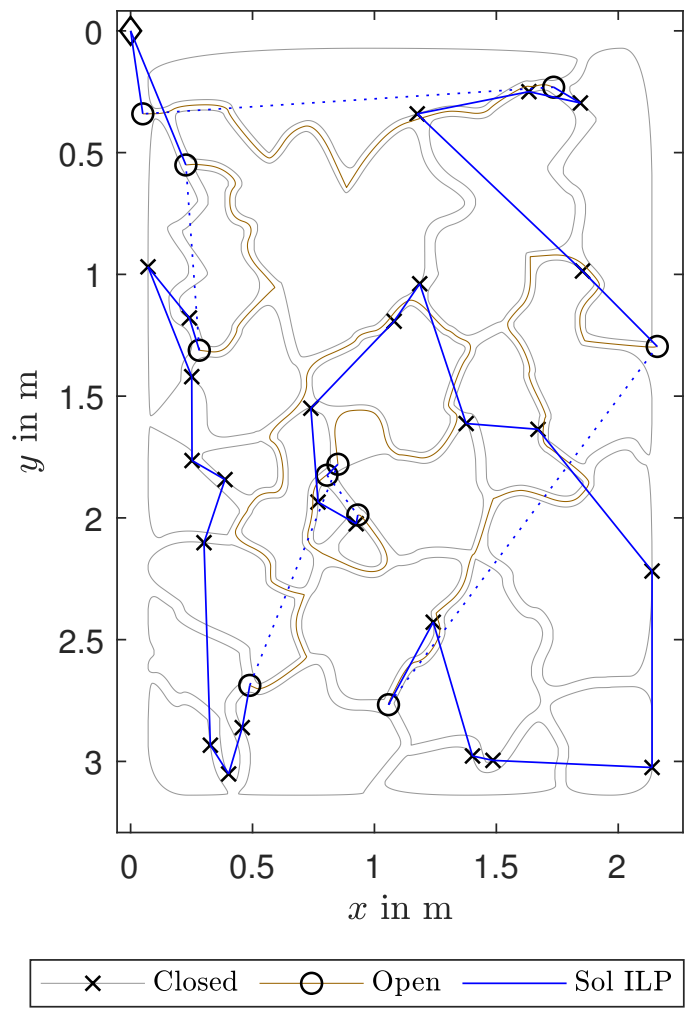

Figure 6. Example $N=31$ : Solution ILP 


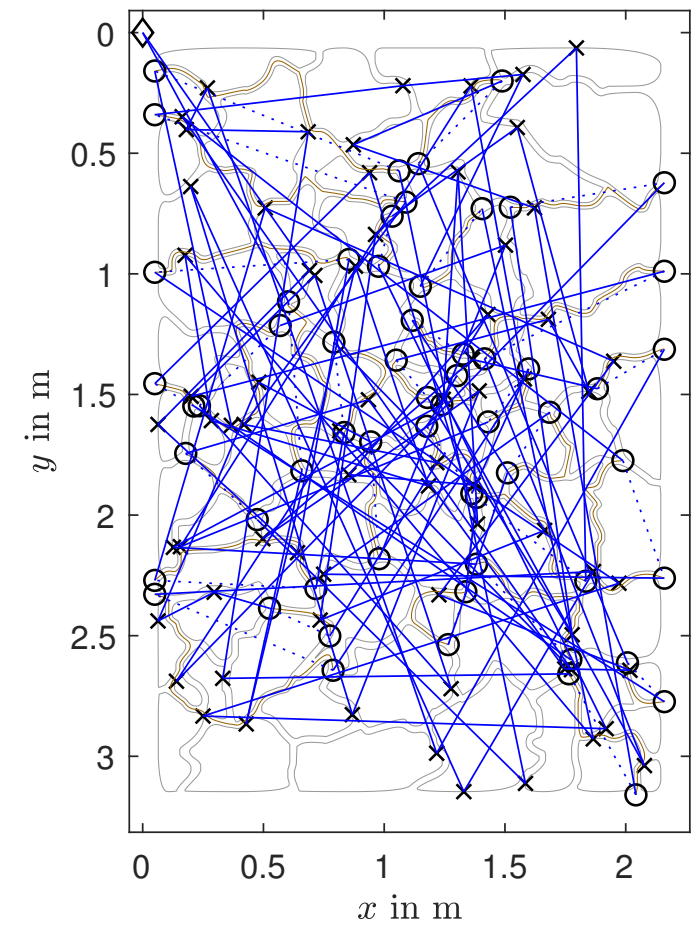

$\times$ Closed O Open $\longrightarrow$ Unsorted

Figure 7. Example $N=101$ : Random Tour

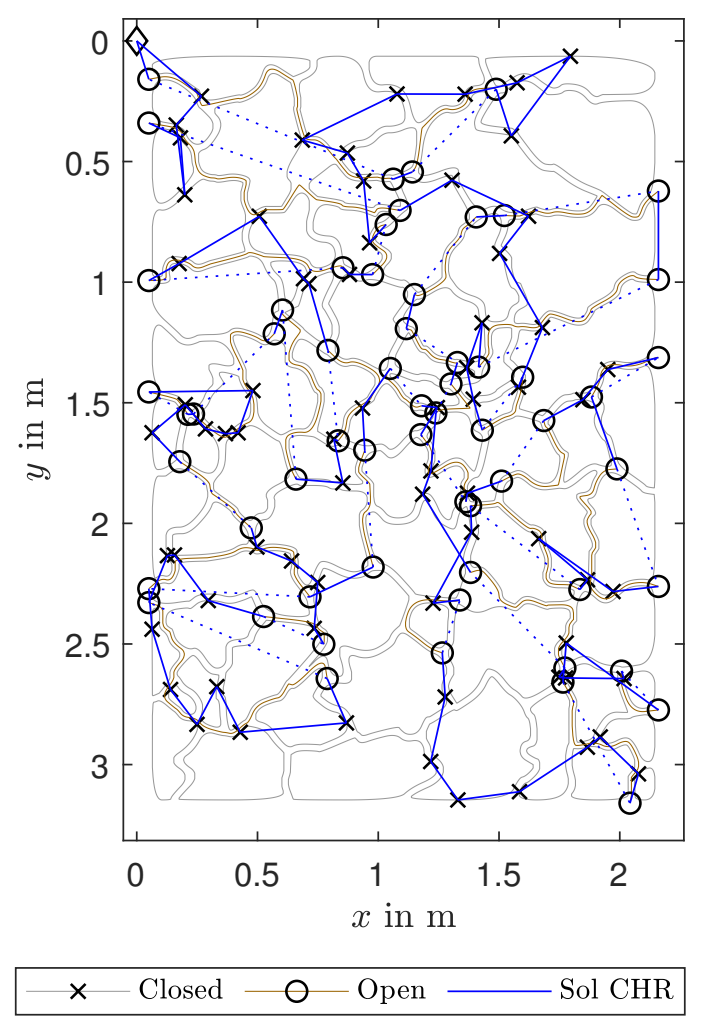

Figure 9. Example $N=101$ : Solution Christofides

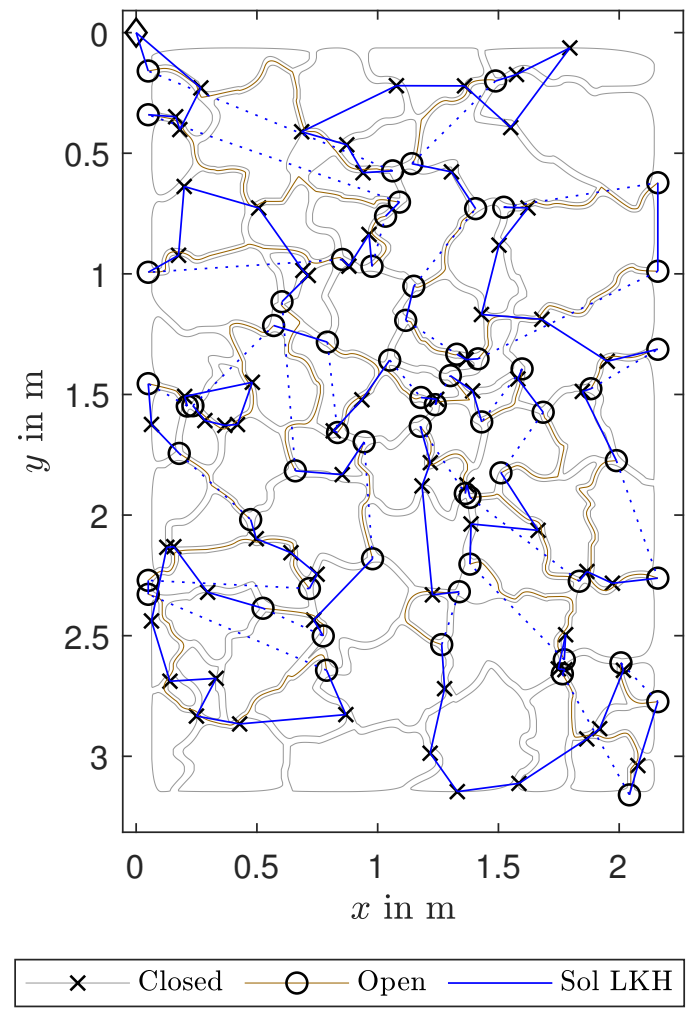

Figure 8. Example $N=101$ : Solution LKH

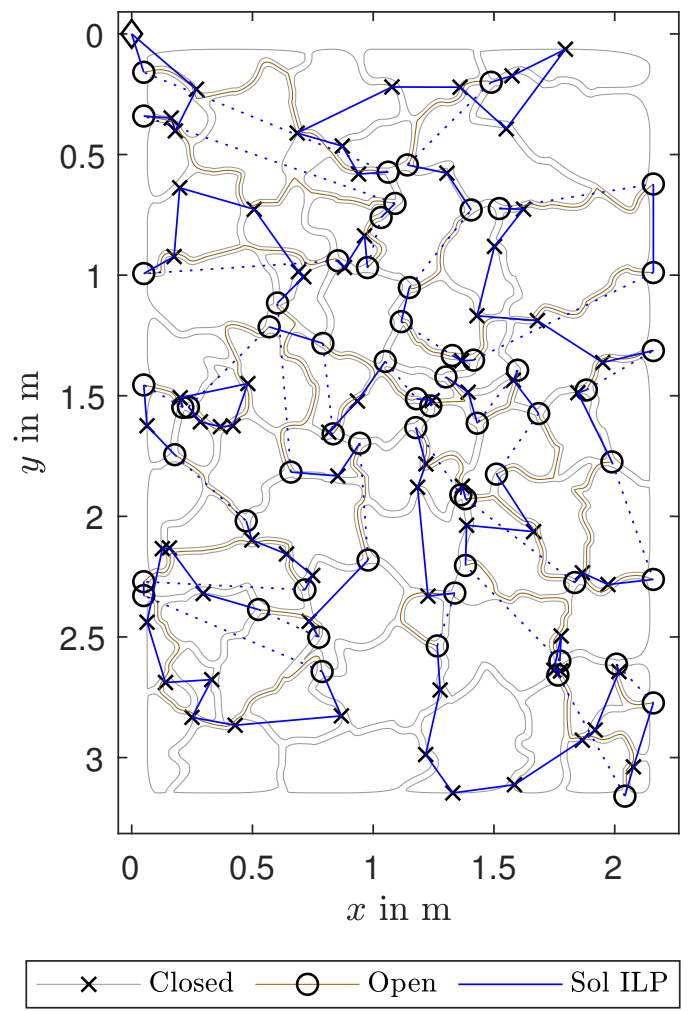

Figure 10. Example $N=101$ : Solution ILP 


\section{CONCLUSION}

It has been shown, that the Christofides heuristic as well as the LKH heuristic can be applied for sequencing open and closed cutting paths, while minimizing the overall traversing time of the nonproductive trajectories, connecting these paths. This has been achieved by minor extensions on the respective algorithms and by introducing a special metric for calculating the edge weights, which models the traversing time of an time optimal $\sin ^{2}$-jerk trajectory along a straight path in the $2 \mathrm{D}$ plane.

Finally the Christofides heuristic, the LKH heuristic and an integer linear programming algorithm are compared, regarding solution quality and calculation time using random generated examples of different problem sizes. The results of these examples indicate that both the Christofides and the LKH heuristic are capable of finding good approximations of the optimal tour for small problem sizes and that the LKH heuristic performs better, especially regarding calculation time, for problems with a higher number of nodes.

\section{ACKNOWLEDGMENTS}

Supported by the "LCM - K2 Center for Symbiotic Mechatronics" within the framework of the Austrian COMET-K2 program.

\section{REFERENCES}

[1] Dewil, R., Vansteenwegen, P., Cattrysse, D.: A review of cutting path algorithms for laser cutters. The International Journal of Advanced Manufacturing Technology 87(5) (2016) 18651884

[2] Dewil, R.: On generating tool paths for laser cutters. (2014)

[3] Christofides, N.: Worst-case analysis of a new heuristic for the travelling salesman problem. Technical report, Carnegie-Mellon Univ Pittsburgh Pa Management Sciences Research Group (1976)

[4] Jörgl, M., Gattringer, H., Müller, A.: An Almost Time Optimal Route Planning Method for Complex Manufacturing Topologies. In: Computer Aided Systems Theory - EUROCAST 2015, Springer International Publishing (2015) 673-680

[5] Helsgaun, K.: An effective implementation of the Lin-Kernighan traveling salesman heuristic. European Journal of Operational Research 126(1) (2000) 106-130

[6] Lin, S., Kernighan, B.W.: An effective heuristic algorithm for the traveling-salesman problem. Operations research 21(2) (1973) 498-516

[7] Dantzig, G., Fulkerson, R., Johnson, S.: Solution of a large-scale traveling-salesman problem. Journal of the operations research society of America 2(4) (1954) 393-410 